Article

\title{
Synthesis and Antifungal Activity of the Derivatives of Novel Pyrazole Carboxamide and Isoxazolol Pyrazole Carboxylate
}

\author{
Jialong Sun and Yuanming Zhou* \\ College of Chemistry and Pharmaceutical Sciences, Qingdao Agricultural University, \\ Qingdao 266109, China; E-Mail: sunjialong6289@163.com \\ * Author to whom correspondence should be addressed; E-Mail: zym7410@163.com; \\ Tel.: +86-532-8608-0147.
}

Academic Editor: Derek J. McPhee

Received: 13 January 2015 / Accepted: 4 March 2015 / Published: 9 March 2015

\begin{abstract}
A series of pyrazole carboxamide and isoxazolol pyrazole carboxylate derivatives were designed and synthesized in this study. The structures of the compounds were elucidated based on spectral data (infrared, proton nuclear magnetic resonance and mass spectroscopy). Then, all of the compounds were bioassayed in vitro against four types of phytopathogenic fungi (Alternaria porri, Marssonina coronaria, Cercospora petroselini and Rhizoctonia solani) using the mycelium growth inhibition method. The results showed that some of the synthesized pyrazole carboxamides displayed notable antifungal activity. The isoxazole pyrazole carboxylate 7ai exhibited significant antifungal activity against $R$. solani, with an $\mathrm{EC}_{50}$ value of $0.37 \mu \mathrm{g} / \mathrm{mL}$. Nonetheless, this value was lower than that of the commercial fungicide, carbendazol.
\end{abstract}

Keywords: pyrazole carboxamide; isoxazolol pyrazole carboxylate; antifungal activity; synthesis; fungi

\section{Introduction}

Phytopathogenic fungi, such as Rhizoctonia solani Kuhn, Alternaria porri (Ell) Ciferri, Marssonina coronaria (Ell.et Davis) Davis and Cercospora petroselini Saccardo, pose serious threats to agriculture. They are broad host-range pathogens and infect many crops worldwide, including rice, onions, apples and cucumbers. Many pesticides have been developed and applied to control these diseases with the 
progression of the modern agrochemical industry. However, the increased microbial resistance of pathogens to known antibiotics facilitates the urgent need for new fungicides [1].

As with many other five-membered heterocyclic compounds, pyrazoles and their derivatives attract increasing attention in the fields of pharmacology and medicine because of their various bioactivities, including antifungal [2], anti-inflammatory [3], antiviral [4], antioxidant [5], cytotoxic [6], antihypertensive [7], A3 adenosine receptor antagonistic [8], antibacterial [9], tranquilizing, psychoanaleptic, muscle-relaxant, hypnotic, antidepressant, ulcerogenic and analgesic activities [10]. They are also highly significant in agrichemistry, and many of these compounds have been widely used, given their fungicidal [11], insecticidal [12] and herbicidal activities [13].

Pyrazole carboxamide derivatives are important heterocyclic compounds in the development of medicines and pesticides because of their broad spectrum of biological activities, including insecticidal [14], fungicidal [15], herbicidal [13] and acaricidal activity [16]. Many recent studies have been conducted on the synthesis and biological activity of these derivatives. Pyrazole carboxamide derivatives, such as penthiopyrad, furametpyr, penflufen, isopyrazam and bixafen, which could inhibit the succinate dehydrogenase, have been developed and commercialized as fungicides in succession [17]. In addition, many isoxazole compounds, including oxacillin and sulfamethoxazole, have been developed as pesticides and drugs, because of their insecticidal, herbicidal, antiviral and antifungal activities. Isoxazole derivatives have received much attention, because of their wide application in medicine and pesticide chemistry [18].

In view of the facts and to explore the potential antifungal activity of pyrazole derivatives, a series of pyrazole carboxamide and isoxazolol pyrazole carboxylate derivatives are designed and synthesized in the current study. Pyrazole carbonyl chloride is synthesized from pyrazole carboxylic acid and thionyl chloride. Then, 18 novel pyrazole carboxamides and two isoxazolol pyrazole carboxylates are synthesized by reacting pyrazole carbonyl chloride with amines and with isoxazol-3-ol, respectively. The structures of all of the synthesized compounds are unequivocally determined through a comprehensive analysis of spectroscopic data from infrared (IR), mass spectroscopy (MS) and proton nuclear magnetic resonance $\left({ }^{1} \mathrm{H}-\mathrm{NMR}\right)$.

\section{Results and Discussion}

\subsection{Chemistry}

The synthesis of the derivatives of pyrazole carboxamide and isoxazolol pyrazole carboxylate is outlined in Scheme 1. Acetoacetic ester $(\mathbf{1 a}-\mathbf{b})$ and triethyl orthoformate were dissolved in acetic anhydride, refluxed and then converted into 2-ethoxymethylene acetoacetic ester derivatives (2a-b) [19]. Ethyl 1H-pyrazole-4-carboxylate (3a-b) was prepared by reacting Compounds $\mathbf{2 a}-\mathbf{b}$ with hydrazine hydrate [20]. Intermediate $1 H$-pyrazole-4-carboxylic acids (5a-b) were obtained as light-yellow crystals from Compounds $\mathbf{3 a}-\mathbf{b}$ by successively performing a substitution reaction using dimethyl sulfate, saponification with $\mathrm{NaOH}$ and acidification using $\mathrm{HCl}$ [21]. Subsequently, Compounds 5a-b were refluxed in $\mathrm{SOCl}_{2}$ to yield pyrazole acid chlorides (6a-b) [22]. Finally, the target compounds of pyrazole carboxamides and isoxazolol pyrazole carboxylates (7aa-bk) were obtained by reacting $\mathbf{6 a}-\mathbf{b}$ with different substituted amines [23] and with isoxazol-3-ol, respectively. 
IR, MS and ${ }^{1} \mathrm{H}-\mathrm{NMR}$ data were applied to confirm the structures of the new synthesized compounds. The synthesized compounds above were published in our Chinese Patents CN103554026A and CN103524417A [24,25].<smiles>[R]C(=O)CC(C)=O</smiles>

$1 a-b$

2a-b

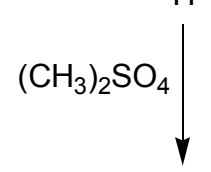

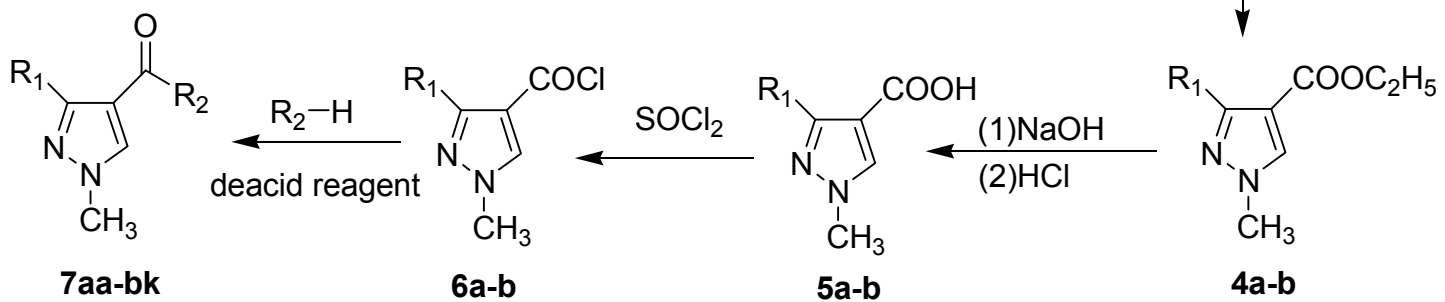

Scheme 1. Synthesis route of the pyrazole derivatives.

\subsection{Antifungal Activity}

The initial concentration was set at $100 \mu \mathrm{g} / \mathrm{mL}$ for antifungal activity screening in vitro. If the percentage inhibition exceeded $50 \%$, a series of concentrations of the compounds was tested to evaluate their EC50 values. Carbendazol was selected as the positive control.

As suggested in Table 1, most of the synthesized pyrazole derivatives exhibited antifungal activity to some extent. Though the EC50 value of these compounds was higher than that of the positive control, carbendazol, a few pyrazole carboxamides (7af, $7 \mathbf{b c}, \mathbf{7 b g}, \mathbf{7 b h}$ and $\mathbf{7 b i}$ ) displayed remarkable antifungal activity. According to the literature [17] reported, a bigger group introduced into the ortho position of the aniline part of this type of compound would strengthen the antifungal activity. The simple anilines applied in this study only gave moderate antifungal activity, so the bioactivity and the structures of the pyrazole derivatives should be researched further.

Table 1. EC50 values of the pyrazole derivatives against four plant pathogenic fungi in vitro.

\begin{tabular}{|c|c|c|c|c|c|c|}
\hline \multirow{2}{*}{ Compound } & \multirow{2}{*}{$\mathbf{R}_{1}$} & \multirow{2}{*}{$\mathbf{R}_{\mathbf{2}}$} & \multicolumn{4}{|c|}{$\mathrm{EC}_{50}(\mu \mathrm{g} / \mathrm{mL})$} \\
\hline & & & A. porri & M. coronaria & C. petroselini & R. solani \\
\hline $7 \mathbf{a a}$ & $\mathrm{CH}_{3}-\{$ & & -- & -- & -- & 31.39 \\
\hline $7 \mathbf{a b}$ & $\mathrm{CH}_{3}-\xi$ & & 80.76 & -- & 38.41 & -- \\
\hline $7 a c$ & $\mathrm{CH}_{3}-\xi$ & & 52.56 & 84.74 & -- & 40.00 \\
\hline
\end{tabular}


Table 1. Cont.

\begin{tabular}{|c|c|c|c|c|c|c|}
\hline \multirow{2}{*}{ Compound } & \multirow{2}{*}{$\mathbf{R}_{\mathbf{1}}$} & \multirow{2}{*}{$\mathbf{R}_{2}$} & \multicolumn{4}{|c|}{$\mathrm{EC}_{50}(\mu \mathrm{g} / \mathrm{mL})$} \\
\hline & & & A. porri & M. coronaria & C. petroselini & R. solani \\
\hline $7 \mathrm{ad}$ & $\mathrm{CH}_{3}-\xi$ & & -- & -- & 6.32 & 18.15 \\
\hline $7 \mathbf{a e}$ & $\mathrm{CH}_{3}-\xi$ & & 65.12 & -- & -- & 14.89 \\
\hline $7 \mathrm{af}$ & $\mathrm{CH}_{3}-\xi$ & & 63.04 & 7.87 & 35.90 & 5.23 \\
\hline $7 \mathrm{ag}$ & $\mathrm{CH}_{3}-\xi$ & & 54.86 & 76.12 & 51.00 & 16.91 \\
\hline $7 \mathbf{a h}$ & $\mathrm{CH}_{3}-\xi$ & & -- & -- & -- & 69.45 \\
\hline $7 \mathbf{a i}$ & $\mathrm{CH}_{3}-\xi$ & & 2.24 & 3.21 & 10.19 & 0.37 \\
\hline $7 \mathbf{b a}$ & $\mathrm{CF}_{3}-\xi$ & & -- & 35.94 & 22.47 & 16.81 \\
\hline $7 \mathbf{b b}$ & $\mathrm{CF}_{3}-\xi$ & & -- & 74.54 & 27.82 & 19.47 \\
\hline $7 \mathrm{bc}$ & $\mathrm{CF}_{3}-\xi$ & & 10.10 & 14.92 & 5.43 & 3.40 \\
\hline $7 b d$ & $\mathrm{CF}_{3}-\xi$ & & -- & -- & 74.38 & 27.37 \\
\hline $7 \mathrm{be}$ & $\mathrm{CF}_{3}-\xi$ & & 23.12 & 13.00 & 13.44 & 8.55 \\
\hline
\end{tabular}


Table 1. Cont.

\begin{tabular}{|c|c|c|c|c|c|c|}
\hline \multirow{2}{*}{ Compound } & \multirow{2}{*}{$\mathbf{R}_{\mathbf{1}}$} & \multirow{2}{*}{$\mathbf{R}_{\mathbf{2}}$} & \multicolumn{4}{|c|}{$\mathrm{EC}_{50}(\mu \mathrm{g} / \mathrm{mL})$} \\
\hline & & & A. porri & M. coronaria & C. petroselini & R. solani \\
\hline $7 \mathrm{bf}$ & $\mathrm{CF}_{3}-\xi$ & & 72.20 & 61.29 & -- & 81.72 \\
\hline $7 b g$ & $\mathrm{CF}_{3}-\xi$ & & 11.22 & 7.93 & 27.43 & 4.99 \\
\hline $7 \mathrm{bh}$ & $\mathrm{CF}_{3}-\{$ & & 24.76 & 25.48 & 6.99 & 5.93 \\
\hline $7 \mathrm{bi}$ & $\mathrm{CF}_{3}-\xi$ & & 21.01 & 9.08 & 32.40 & 7.69 \\
\hline $7 \mathbf{b j}$ & $\mathrm{CF}_{3}-\xi$ & & 11.46 & 15.86 & -- & 8.32 \\
\hline $7 \mathbf{b k}$ & $\mathrm{CF}_{3}-\xi$ & & 35.05 & -- & -- & 28.88 \\
\hline carbendazol & & & 0.99 & 0.96 & 0.96 & 1.00 \\
\hline
\end{tabular}

“--" The percentage of inhibition is lower than $50 \%$ at $100 \mu \mathrm{g} / \mathrm{mL}$.

It was interesting that the isoxazolol pyrazole carboxylate $7 \mathbf{a i}$ showed significant antifungal activity against $A$. porri, M. coronaria, C. petroselini and $R$. solani, with $\mathrm{EC}_{50}$ values of 2.24, 3.21, 10.29 and $0.37 \mu \mathrm{g} / \mathrm{mL}$, respectively. The $\mathrm{EC}_{50}$ value of this compound against $R$. solani was lower than that of the positive control, carbendazol (EC50, $1.00 \mu \mathrm{g} / \mathrm{mL})$.

The $\mathrm{EC}_{50}$ values of the isoxazolol pyrazole carboxylate $7 \mathbf{b k}$ were 35.05 , over 100 , over 100 and $28.88 \mu \mathrm{g} / \mathrm{mL}$ against A. porri, M. coronaria, C. petroselini and R. solani, respectively (Table 1). The results of the preliminary structure-activity relationship (SAR) analysis suggested that the antifungal activity of the synthesized isoxazolol pyrazole carboxylate was significantly weakened when the methyl group at the C-3 of the pyrazole ring (7ai) was substituted with a trifluoromethyl group (7bk).

\section{Experimental Section}

\subsection{Chemistry}

All of the reagents and solvents were either chemically pure or purified in accordance with standard methods. Reactions were monitored through thin-layer chromatography (TLC) using precoated silica gel plates (silica gel GF 254, Qingdao Marine Chemistry Co. Ltd., Qingdao, China), and the spots 
were visualized with UV $(254 \mathrm{~nm})$. All of the melting points were detected with a WRS-1A type melting point apparatus (ShangHai Suoguang Electric Tech Co., Ltd., Shanghai, China), and the thermometer was not corrected. IR spectra were recorded on a Nicolet IR-200 (Thermo Electron, Madison, WI, USA) spectrophotometer. ${ }^{1} \mathrm{H}-\mathrm{NMR}$ spectra were captured with Bruker AV-500 and AV-400 spectrometers, and tetramethylsilane was applied as an internal standard. High-resolution electrospray ionization mass spectroscopy (HR-ESI-MS) spectra were observed with a Micromass Q-TOF spectrometer (Waters Corp., Manchester, UK).

\subsection{General Procedure for the Preparation of $\mathbf{2 a - b}$}

A mixture of acetoacetic ester $(0.60 \mathrm{~mol})(\mathbf{1 a}-\mathbf{b})$, triethyl orthoformate $(0.72 \mathrm{~mol})$ and acetic anhydride (1.08 mol) was stirred and heated under reflux until the $\mathbf{1 a}-\mathbf{b}$ was no longer monitored by TLC. Then, the reaction mixture was evaporated in vacuo. The distillates of $\mathrm{T}=140{ }^{\circ} \mathrm{C}-160{ }^{\circ} \mathrm{C}$ $(3 \mathrm{KPa})$ were collected to produce 2-ethoxymethylene acetoacetic esters $(\mathbf{2 a}-\mathbf{b})$ as a light-yellow liquid with yields ranging from $70 \%$ to $90 \%$.

\subsection{General Procedure for the Preparation of $\mathbf{3 a}-\mathbf{b}$}

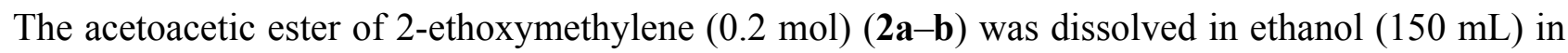
an ice-water bath, and $80 \%$ hydrazine hydrate $(0.4 \mathrm{~mol})$ was added dropwise. The mixture reacted at room temperature until the $\mathbf{2 a}-\mathbf{b}$ was fully consumed, as detected by TLC. Subsequently, the reaction mixture was concentrated in vacuo. The residue was extracted with 1,2-dichloroethane, washed with water and brine, dried over anhydrous sodium sulfate and then concentrated in vacuo to obtain ethyl $1 H$-pyrazole-4-carboxylate (3a-b) as either light-yellow liquids or solids.

\subsection{General Procedure for the Preparation of 5a-b}

Ethyl $1 \mathrm{H}$-pyrazole-4-carboxylate $(3 \mathbf{a}-\mathbf{b}, 0.5 \mathrm{~mol})$ and $\mathrm{NaHCO}_{3}(0.6 \mathrm{~mol})$ were dissolved in toluene $(120 \mathrm{~mL}) .\left(\mathrm{CH}_{3}\right)_{2} \mathrm{SO}_{4}(0.24 \mathrm{~mol})$ was dropped gradually into the solution while the temperature was maintained at $20^{\circ} \mathrm{C}-30{ }^{\circ} \mathrm{C}$ via an ice-water bath. Then, the solution reacted at a temperature of $50{ }^{\circ} \mathrm{C}$ with a water bath and was monitored by TLC. Once the reaction was complete, the reaction solution was filtered. A light-yellow solution was then obtained and washed with ice water. The upper toluene solution was concentrated in vacuo to produce ethyl 1-methyl-1H-pyrazole-4-carboxylate (4a-b). Subsequently, sodium hydroxide solution $(0.12 \mathrm{~mol} \mathrm{NaOH}$ dissolved in $45 \mathrm{~mL}$ water $)$ was added to a solution of Compounds $4 \mathbf{a}-\mathbf{b}(0.1 \mathrm{~mol})$ in $\mathrm{EtOH}(95 \%, 50 \mathrm{~mL})$ and then reacted at room temperature for approximately $2 \mathrm{~h}$. The solution was concentrated in vacuo to remove most of the ethanol. The $\mathrm{pH}$ level was then adjusted to 3-4 with $\mathrm{HCl}$. The reaction mixture was filtered, and the filtrate was recrystallized with ethyl acetate to produce pyrazole acids $(\mathbf{5 a}-\mathbf{b})$ as light-yellow crystalline solids.

\subsection{General Procedure for the Preparation of $\mathbf{7 a a}-\mathbf{b k}$}

Pyrazole acid chlorides $\mathbf{6 a}-\mathbf{b}$ were prepared by refluxing $\mathbf{4 a}-\mathbf{b}$ in thionyl chloride for $8 \mathrm{~h}$. Pyrazole acid chlorides $\mathbf{6 a}-\mathbf{b}(12 \mathrm{mmol})$ in anhydrous tetrahydrofuran (THF; $30 \mathrm{~mL}$ ) were slowly added to a solution of amine derivatives or 5-methylisoxazol-3-ol $(10 \mathrm{mmol})$ and $\mathrm{K}_{2} \mathrm{CO}_{3}(1.38 \mathrm{~g}$, $10 \mathrm{mmol})$ in 
anhydrous THF $(30 \mathrm{~mL})$ at a controlled temperature of $5{ }^{\circ} \mathrm{C}$. The reaction proceeded at room temperature until $\mathbf{6 a}-\mathbf{b}$ was no longer tested by TLC. The reaction solution was then filtered and the solvent distilled. The residue was dissolved in ethyl acetate, washed with water and brine, dried over anhydrous sodium sulfate and recrystallized to generate the target pyrazole carboxamides and isoxazolol pyrazole carboxylates (7aa-bk). The product yields ranged from $40 \%$ to $80 \%$. All 20 compounds were novel, and the physical and spectral data for these compounds are listed below.

1,3-Dimethyl-N-(2-hydroxyl)benzyl-1H-pyrazole-4-carboxamide (7aa): Bright brown crystal, yield of 77.9\%, m.p. $193.0-193.1{ }^{\circ} \mathrm{C} .{ }^{1} \mathrm{H}-\mathrm{NMR}\left(\mathrm{CDCl}_{3}, 500 \mathrm{MHz}\right) \delta: 8.82(\mathrm{~s}, 1 \mathrm{H}, \mathrm{NH}), 7.86(\mathrm{~s}, 1 \mathrm{H}$, pyrazole H), 7.61 (s, 1H, -OH), 7.14-7.16 (m, 1H, Ar-H), 7.13-7.09 (m, 1H, Ar-H), 7.08-7.05 (m, 1H, Ar-H), 6.89 (t, $J=1.5 \mathrm{~Hz}, 1 \mathrm{H}, \mathrm{Ar}-\mathrm{H}), 3.89$ (s, 3H, N-CH3), 2.56 (s, 3H, pyrazole $\mathrm{CH}_{3}$ ); IR (KBr): $v$ 3431, 1643, 1593, 1544, 1452, 1382, $1278 \mathrm{~cm}^{-1}$; HR-ESI-MS m/z: $232.1084[\mathrm{M}+\mathrm{H}]^{+}$(calcd. for $\left.\mathrm{C}_{12} \mathrm{H}_{14} \mathrm{~N}_{3} \mathrm{O}_{2}, 232.1081\right)$.

1,3-Dimethyl-N-(4-hydroxyl)benzyl-1H-pyrazole-4-carboxamide (7ab): Gray needle crystal, yield of 80.5\%, m.p. 208.0-208.7 ${ }^{\circ} \mathrm{C} .{ }^{1} \mathrm{H}-\mathrm{NMR}\left(\mathrm{CDCl}_{3}, 500 \mathrm{MHz}\right) \delta: 7.76$ (s, 1H, pyrazole H), 7.43 (m, 2H, Ar-H), $6.84(\mathrm{~d}, J=8.5 \mathrm{~Hz}, 2 \mathrm{H}, \operatorname{Ar}-\mathrm{H}), 4.78(\mathrm{~s}, 1 \mathrm{H},-\mathrm{OH}), 3.89\left(\mathrm{~s}, 3 \mathrm{H}, \mathrm{N}-\mathrm{CH}_{3}\right), 2.54(\mathrm{~s}, 3 \mathrm{H}$, pyrazole $\left.\mathrm{CH}_{3}\right)$; IR (KBr): v 3510, 3290, 1639, 1539, 1515, 1448, 1245, $1166 \mathrm{~cm}^{-1}$; HR-ESI-MS m/z: 232.1081 [M+H] $]^{+}$calcd. for $\mathrm{C}_{12} \mathrm{H}_{14} \mathrm{~N}_{3} \mathrm{O}_{2}, 232.1081$ ).

1,3-Dimethyl-N-2',4'-dichlorobenzyl-1H-pyrazole-4-carboxamide (7ac): White lamellar crystal, yield of 77.9\%, m.p. $169.1-169.3{ }^{\circ} \mathrm{C} .{ }^{1} \mathrm{H}-\mathrm{NMR}\left(\mathrm{CDCl}_{3}, 500 \mathrm{MHz}\right) \delta: 8.52(\mathrm{~d}, J=9.0 \mathrm{~Hz}, 1 \mathrm{H}, \operatorname{Ar}-\mathrm{H})$, 7.86 (s, 1H, pyrazole H), 7.42 (d, J=2.5 Hz, 1H, Ar-H), 7.27-7.30 (m, 1H, Ar-H), 3.91 (s, 3H, N-CH3), 2.60 (s, 3H, pyrazole $\mathrm{CH}_{3}$ ); IR (KBr): $v$ 3241, 1648, 1499, 1279, $1096 \mathrm{~cm}^{-1}$; HR-ESI-MS m/z: 284.0347 [M+H] $]^{+}$(calcd. for $\mathrm{C}_{12} \mathrm{H}_{12} \mathrm{Cl}_{2} \mathrm{~N}_{3} \mathrm{O}, 284.0352$ ).

1,3-Dimethyl-N-3',5'-dichlorobenzyl-1H-pyrazole-4-carboxamide (7ad): Gray crystal, yield of 66.9\%, m.p. 181.6-182.2 ${ }^{\circ} \mathrm{C} .{ }^{1} \mathrm{H}-\mathrm{NMR}\left(\mathrm{CDCl}_{3}, 500 \mathrm{MHz}\right) \delta$ : 7.77 (s, 1H, pyrazole $\left.\mathrm{H}\right), 7.54(\mathrm{~d}, J=1.5 \mathrm{~Hz}, 2 \mathrm{H}$, Ar-H), $7.11(\mathrm{~s}, 1 \mathrm{H}, \mathrm{Ar}-\mathrm{H}), 3.88\left(\mathrm{~s}, 3 \mathrm{H}, \mathrm{N}-\mathrm{CH}_{3}\right), 2.53$ (s, 3H, pyrazole $\left.\mathrm{CH}_{3}\right)$; IR (KBr): v 3265, $3211,1698,1614,1503,1403,1142,1063 \mathrm{~cm}^{-1}$; HR-ESI-MS m/z: $284.0357[\mathrm{M}+\mathrm{H}]^{+}$(calcd. for $\left.\mathrm{C}_{12} \mathrm{H}_{12} \mathrm{Cl}_{2} \mathrm{~N}_{3} \mathrm{O}, 284.0352\right)$.

1,3-Dimethyl-N-(1,3,4-thiadiazole-2-yl)-1H-pyrazole-4-carboxamide (7ae): White lamellar crystal, yield of 57.2\%, m.p. 278.4-278.9 ${ }^{\circ} \mathrm{C} .{ }^{1} \mathrm{H}-\mathrm{NMR}$ (DMSO- $\left.d 6,500 \mathrm{MHz}\right) \delta: 12.52$ (s, 1H, NH), 9.15 (s, $1 \mathrm{H}$, pyrazole $\mathrm{H}), 8.55(\mathrm{~s}, 1 \mathrm{H}$, thiadiazole $\mathrm{H}), 3.82\left(\mathrm{~s}, 3 \mathrm{H}, \mathrm{N}-\mathrm{CH}_{3}\right), 2.50\left(\mathrm{~s}, 3 \mathrm{H}\right.$, pyrazole $\left.\mathrm{CH}_{3}\right)$; IR (KBr): $v$ 3381, 3124, 2929, 1680, 1548, 1415, $1311 \mathrm{~cm}^{-1}$; HR-ESI-MS m/z: $224.0597[\mathrm{M}+\mathrm{H}]^{+}$(calcd. for $\left.\mathrm{C}_{8} \mathrm{H}_{10} \mathrm{~N}_{5} \mathrm{OS}, 224.0601\right)$.

1,3-Dimethyl-N-(5-ethyl-1,3,4-thiadiazole-2-yl)-1H-pyrazole-4-carboxamide (7af): White powder, yield of $68.3 \%$, m.p. $266.2-266.5{ }^{\circ} \mathrm{C} .{ }^{1} \mathrm{H}-\mathrm{NMR}\left(\mathrm{CDCl}_{3}, 400 \mathrm{MHz}\right) \delta$ : 12.58 (s, 1H, NH), $9.13(\mathrm{~s}, 1 \mathrm{H}$, pyrazole H), 3.94 (s, 3H, N-CH3), 3.07 (q, $\left.J=1.0 \mathrm{~Hz}, 2 \mathrm{H}, \mathrm{CH}_{2}\right), 2.56$ (s, 3H, pyrazole $\mathrm{CH}_{3}$ ), 1.45 (t, $\left.J=1.0 \mathrm{~Hz}, 3 \mathrm{H}, \mathrm{CH}_{3}\right)$; IR (KBr): v 3129, 2971, 2934, 1673, 1544, 1420, 1316, 1241, $1179 \mathrm{~cm}^{-1}$; HR-ESI-MS $m / z$ : $252.0912[\mathrm{M}+\mathrm{H}]^{+}$(calcd. for $\mathrm{C}_{10} \mathrm{H}_{14} \mathrm{~N}_{5} \mathrm{OS}, 252.0914$ ). 
1,3-Dimethyl-N-(5-methyl-1,3,4-thiadiazole-2-yl)-1H-pyrazole-4-carboxamide (7ag): White powder, yield of $61.4 \%$, m.p. $>300{ }^{\circ} \mathrm{C} .{ }^{1} \mathrm{H}-\mathrm{NMR}\left(\mathrm{CDCl}_{3}, 400 \mathrm{MHz}\right) \delta: 9.12(\mathrm{~s}, 1 \mathrm{H}$, pyrazole $\mathrm{H}), 3.96(\mathrm{~s}, 3 \mathrm{H}$, $\left.\mathrm{N}-\mathrm{CH}_{3}\right), 2.72\left(\mathrm{~s}, 3 \mathrm{H}\right.$, thiadiazole $\left.\mathrm{CH}_{3}\right), 2.56\left(\mathrm{~s}, 3 \mathrm{H}\right.$, pyrazole $\left.\mathrm{CH}_{3}\right)$; IR (KBr): $v$ 3149, 3012, 2921, 1677, 1544, 1494, 1416, 1320, 1250, $1188 \mathrm{~cm}^{-1}$; HR-ESI-MS m/z: $238.0755[\mathrm{M}+\mathrm{H}]^{+}$(calcd. for $\left.\mathrm{C}_{9} \mathrm{H}_{12} \mathrm{~N}_{5} \mathrm{OS}, 238.0757\right)$.

1,3-Dimethyl-N-(5-trifluoromethyl-1,3,4-thiadiazole-2-yl)-1H-pyrazole-4-carboxamide (7ah): White lamellar crystal, yield of $44.3 \%$, m.p. $272.1-272.8{ }^{\circ} \mathrm{C} .{ }^{1} \mathrm{H}-\mathrm{NMR}\left(\mathrm{CDCl}_{3}, 500 \mathrm{MHz}\right) \delta: 12.61(\mathrm{~s}, 1 \mathrm{H}$, $\mathrm{NH}), 9.12$ (s, 1H, pyrazole H), $3.98\left(\mathrm{~s}, 3 \mathrm{H}, \mathrm{N}-\mathrm{CH}_{3}\right), 2.58$ (s, 3H, pyrazole $\left.\mathrm{CH}_{3}\right)$; IR $(\mathrm{KBr}): v 1668,1519$, 1303, 1195, 1137, $1029 \mathrm{~cm}^{-1}$; HR-ESI-MS m/z: $292.0471[\mathrm{M}+\mathrm{H}]^{+}$(calcd. for $\mathrm{C}_{9} \mathrm{H}_{9} \mathrm{~F}_{3} \mathrm{~N}_{5} \mathrm{OS}, 292.0474$ ).

5-Methylisoxazol-3-yl 1,3-dimethyl-1H-pyrazole-4-carboxylate (7ai): White powder, yield of 54.7\%, m.p. $124.2-124.4{ }^{\circ} \mathrm{C} .{ }^{1} \mathrm{H}-\mathrm{NMR}\left(\mathrm{CDCl}_{3}, 500 \mathrm{MHz}\right) \delta$ : $7.98(\mathrm{~s}, 1 \mathrm{H}$, pyrazole $\mathrm{H}), 6.23(\mathrm{~s}, 1 \mathrm{H}$, isoxazole $\mathrm{H})$, $3.88\left(\mathrm{~s}, 3 \mathrm{H}, \mathrm{N}-\mathrm{CH}_{3}\right), 2.49$ (s, 3H, pyrazole $\left.\mathrm{CH}_{3}\right), 2.43$ (s, 3H, isoxazole $\left.\mathrm{CH}_{3}\right)$; IR ( $\left.\mathrm{KBr}\right)$ : v 3120, 1743, 1622, 1548, 1232, $1050 \mathrm{~cm}^{-1}$; HR-ESI-MS m/z: $222.0871[\mathrm{M}+\mathrm{H}]^{+}$(calcd. for $\mathrm{C}_{10} \mathrm{H}_{12} \mathrm{~N}_{3} \mathrm{O}_{3}, 222.0873$ ).

1-Methyl-N-(2-hydroxyl)benzyl-3-(trifluoromethyl)-1H-pyrazole-4-carboxamide (7ba): Light yellow crystal, yield of $41.8 \%$, m.p. $192.8-193.2{ }^{\circ} \mathrm{C} .{ }^{1} \mathrm{H}-\mathrm{NMR}$ (DMSO-d6, $\left.500 \mathrm{MHz}\right) \delta: 9.24$ (s, 1H, -OH), 8.54 (s, 1H, pyrazole H), 7.65 (d, $J=7.5 \mathrm{~Hz}, 1 \mathrm{H}, \mathrm{Ar}-\mathrm{H}), 6.98$ (d, $J=7.5 \mathrm{~Hz}, 1 \mathrm{H}, \mathrm{Ar}-\mathrm{H}), 6.88$ (d, $J=7.5 \mathrm{~Hz}, 1 \mathrm{H}, \mathrm{Ar}-\mathrm{H}), 6.79(\mathrm{~d}, J=8.0 \mathrm{~Hz}, 1 \mathrm{H}, \mathrm{Ar}-\mathrm{H}), 3.94\left(\mathrm{~s}, 3 \mathrm{H}, \mathrm{N}-\mathrm{CH}_{3}\right)$; IR (KBr): v 3485, 3116, 1656, 1598, 1494, 1441, 1329, 1250, 1175, $1146 \mathrm{~cm}^{-1}$; HR-ESI-MS m/z: $286.0804[\mathrm{M}+\mathrm{H}]^{+}$(calcd. for $^{2}$ $\left.\mathrm{C}_{12} \mathrm{H}_{11} \mathrm{~F}_{3} \mathrm{~N}_{3} \mathrm{O}_{2}, 286.0798\right)$.

1-Methyl-N-(4-hydroxyl)benzyl-3-(trifluoromethyl)-1H-pyrazole-4-carboxamide (7bb): light brown powder, yield of 67.9\%, m.p. 206.4-206.6 ${ }^{\circ} \mathrm{C} .{ }^{1} \mathrm{H}-\mathrm{NMR}$ (DMSO- $\left.d_{6}, 500 \mathrm{MHz}\right) \delta: 9.88$ (s, 1H, NH), 9.25 (s, 1H, -OH), 8.44 (s, 1H, pyrazole H), 7.38-7.43 (m, 2H, Ar-H), 6.69-6.72 (m, 2H, Ar-H), 3.98 $\left(\mathrm{s}, 3 \mathrm{H}, \mathrm{N}-\mathrm{CH}_{3}\right)$; IR (KBr): $v$ 1627, 1553, 1436, 1329, 1142, 839, 781, 711, 619, $516 \mathrm{~cm}^{-1}$; HR-ESI-MS $m / z$ : $286.0800[\mathrm{M}+\mathrm{H}]^{+}$(calcd. for $\mathrm{C}_{12} \mathrm{H}_{11} \mathrm{~F}_{3} \mathrm{~N}_{3} \mathrm{O}_{2}, 286.0798$ ).

1-Methyl-N-2',4'-dichlorobenzyl-3-(trifluoromethyl)-1H-pyrazole-4-carboxamide (7bc): White crystal, yield of $49.0 \%$, m.p. $147.4-147.8{ }^{\circ} \mathrm{C} .{ }^{1} \mathrm{H}-\mathrm{NMR}\left(\mathrm{CDCl}_{3}, 400 \mathrm{MHz}\right) \delta: 8.43(\mathrm{~d}, J=7.2 \mathrm{~Hz}, 1 \mathrm{H}, \mathrm{Ar}-\mathrm{H})$, 8.04 (s, 1H, pyrazole H), 7.43 (d, $J=2.0 \mathrm{~Hz}, 1 \mathrm{H}$, Ar-H), 7.26-7.30 (m, overlapped, Ar-H), 4.02 (s, 3H, $\left.\mathrm{N}-\mathrm{CH}_{3}\right)$; IR (KBr): $v 2958,1785,1640,1565,1424,1333,1291,1146,1047,847,781,706 \mathrm{~cm}^{-1}$; HR-ESI-MS $m / z$ : $338.0066[\mathrm{M}+\mathrm{H}]^{+}$(calcd. for $\mathrm{C}_{12} \mathrm{H}_{9} \mathrm{Cl}_{2} \mathrm{~F}_{3} \mathrm{~N}_{3} \mathrm{O}, 338.0069$ ).

1-Methyl-N-3',5'-dichlorobenzyl-3-(trifluoromethyl)-1H-pyrazole-4-carboxamide (7bd): White powder, yield of 53.2\%, m.p. 149.1-150.3 ${ }^{\circ} \mathrm{C} .{ }^{1} \mathrm{H}-\mathrm{NMR}\left(\mathrm{CDCl}_{3}, 500 \mathrm{MHz}\right) \delta: 8.42(\mathrm{~d}, J=9.0 \mathrm{~Hz}, \mathrm{Ar}-\mathrm{H})$, 8.03 (s, 1H, pyrazole H), 7.42 (d, $J=2.0 \mathrm{~Hz}, 1 \mathrm{H}, \mathrm{Ar}-\mathrm{H}), 7.26-7.30$ (m, overlapped, Ar-H), 4.01 (s, 3H, $\left.\mathrm{N}-\mathrm{CH}_{3}\right)$; IR (KBr): $v$ 3299, 3162, 3100, 1669, 1582, 1523, 1494, 1461, 1387, $1304 \mathrm{~cm}^{-1}$; HR-ESI-MS m/z: $338.0075[\mathrm{M}+\mathrm{H}]^{+}$(calcd. for $\mathrm{C}_{12} \mathrm{H}_{9} \mathrm{Cl}_{2} \mathrm{~F}_{3} \mathrm{~N}_{3} \mathrm{O}, 338.0069$ ).

1-Methyl-N-(1,3,4-thiadiazole-2-yl)-3-(trifluoromethyl)-1H-pyrazole-4-carboxamide (7be): White floccus, yield of 49.6\%, m.p. $>300{ }^{\circ} \mathrm{C}$. ${ }^{1} \mathrm{H}-\mathrm{NMR}$ (DMSO-d6, $500 \mathrm{MHz}$ ) $\delta: 12.99$ (s, 1H, NH), 9.21 (s, $1 \mathrm{H}$, thiadiazole $\mathrm{H}), 8.76$ (s, 1H, pyrazole $\mathrm{H}), 3.99$ (s, 3H, N-CH3); IR (KBr): v 3170, 3116, 3054, 1689, 
1557, 1436, 1316, 1175, 1134, 1059, 1005, $872 \mathrm{~cm}^{-1}$; HR-ESI-MS m/z: $278.0320[\mathrm{M}+\mathrm{H}]^{+}$(calcd. for $\left.\mathrm{C}_{8} \mathrm{H}_{7} \mathrm{~F}_{3} \mathrm{~N}_{5} \mathrm{OS}, 278.0318\right)$.

1-Methyl-N-(5-ethyl-1,3,4-thiadiazole-2-yl)-3-(trifluoromethyl)-1H-pyrazole-4-carboxamide

(7bf):

White powder, yield of 56.9\%, m.p. 288.6-288.8 ${ }^{\circ} \mathrm{C} .{ }^{1} \mathrm{H}-\mathrm{NMR}$ (DMSO-d6, $\left.500 \mathrm{MHz}\right) \delta: 12.82(\mathrm{~s}, 1 \mathrm{H}$, $\mathrm{NH}), 8.72$ (s, 1H, pyrazole H), $3.96\left(\mathrm{~s}, 3 \mathrm{H}, \mathrm{N}-\mathrm{CH}_{3}\right), 2.98$ (q, $\left.J=7.5 \mathrm{~Hz}, 2 \mathrm{H}, \mathrm{CH}_{2}\right), 1.29$ (t, $J=7.5 \mathrm{~Hz}$, $\left.3 \mathrm{H}, \mathrm{CH}_{3}\right)$; IR (KBr): $v$ 3456, 3054, 2963, 1694, 1627, 1557, 1424, 1341, 1308, 1171, $1129 \mathrm{~cm}^{-1}$; HR-ESI-MS $m / z$ : $306.0631[\mathrm{M}+\mathrm{H}]^{+}$(calcd. for $\mathrm{C}_{10} \mathrm{H}_{11} \mathrm{~F}_{3} \mathrm{~N}_{5} \mathrm{OS}, 306.0631$ ).

1-Methyl-N-(5-trifluoromethyl-1,3,4-thiadiazole-2-yl)-3-(trifluoromethyl)-1H-pyrazole-4-carboxamide

(7bg): White crystal, yield of 71.2\%, m.p. 234.9-235.1 ${ }^{\circ} \mathrm{C} .{ }^{1} \mathrm{H}-\mathrm{NMR}$ (DMSO-d6, $500 \mathrm{MHz}$ ) $\delta: 13.68$ (s, 1H, NH), 8.81 (s, 1H, pyrazole H), 4.01 (s, 3H, N-CH3); IR (KBr): v 3456, 3070, 2963, 1694, $1627,1557,1424,1341,1308,1183,885,711 \mathrm{~cm}^{-1}$; HR-ESI-MS $m / z: 346.0191[\mathrm{M}+\mathrm{H}]^{+}$(calcd. for $\left.\mathrm{C}_{9} \mathrm{H}_{6} \mathrm{~F}_{6} \mathrm{~N}_{5} \mathrm{OS}, 346.0192\right)$.

1-Methyl-N-2'-fluorobenzyl-3-(trifluoromethyl)-1H-pyrazole-4-carboxamide (7bh): Brown crystal, yield of $74.3 \%$, m.p. $148.4 \sim 148.9{ }^{\circ} \mathrm{C} .{ }^{1} \mathrm{H}-\mathrm{NMR}\left(\mathrm{CDCl}_{3}, 500 \mathrm{MHz}\right) \delta: 8.38(\mathrm{~m}, 1 \mathrm{H}, \mathrm{Ar}-\mathrm{H}), 8.03(\mathrm{~s}, 1 \mathrm{H}$, pyrazole H), 8.00-8.03 (m, 1H, Ar-H), 7.08-7.18 (m, 2H, Ar-H), 4.00 (s, 3H, N-CH3); IR (KBr): $v$ 3245, 1652, 1565, 1507, 1441, 1316, 1217, $1183 \mathrm{~cm}^{-1}$; HR-ESI-MS m/z: $288.0757[\mathrm{M}+\mathrm{H}]^{+}$(calcd. for $\mathrm{C}_{12} \mathrm{H}_{10} \mathrm{~F}_{4} \mathrm{~N}_{3} \mathrm{O}, 288.0755$ ).

1-Methyl-N-3'-fluorobenzyl-3-(trifluoromethyl)-1H-pyrazole-4-carboxamide (7bi): Brown crystal, yield of 47.9\%, m.p. $137.8-138.3{ }^{\circ} \mathrm{C} .{ }^{1} \mathrm{H}-\mathrm{NMR}\left(\mathrm{CDCl}_{3}, 500 \mathrm{MHz}\right) \delta: 8.04$ (s, 1H, pyrazole H), 7.76 (s, $1 \mathrm{H}, \mathrm{Ar}-\mathrm{H}), 7.58$ (d, $J=10.5 \mathrm{~Hz}, 1 \mathrm{H}, \mathrm{Ar}-\mathrm{H}), 7.18$ (d, $J=8.5 \mathrm{~Hz}, 1 \mathrm{H}, \mathrm{Ar}-\mathrm{H}), 6.87$ (t, $J=8.5 \mathrm{~Hz}, 1 \mathrm{H}$, Ar-H), 4.01 (s, 3H, N-CH3); IR (KBr): v 3494, 3373, 3261, 1664, 1602, 1561, 1329, 1300, $1188 \mathrm{~cm}^{-1}$; HR-ESI-MS $m / z$ : $288.0757[\mathrm{M}+\mathrm{H}]^{+}$(calcd. for $\mathrm{C}_{12} \mathrm{H}_{10} \mathrm{~F}_{4} \mathrm{~N}_{3} \mathrm{O}, 288.0755$ ).

1-Methyl-N-3',4'-difluorobenzyl-3-(trifluoromethyl)-1H-pyrazole-4-carboxamide (7bj): Light brown crystal, yield of $63.2 \%$, m.p. $155.7-155.8{ }^{\circ} \mathrm{C} .{ }^{1} \mathrm{H}-\mathrm{NMR}\left(\mathrm{CDCl}_{3}, 400 \mathrm{MHz}\right) \delta: 8.04$ (s, 1H, pyrazole H), 7.78 (s, 1H, Ar-H), 7.69 (d, $J=7.5 \mathrm{~Hz}, 1 \mathrm{H}, \operatorname{Ar}-\mathrm{H}), 7.15$ (d, $J=7.0 \mathrm{~Hz}, 1 \mathrm{H}, \mathrm{Ar}-\mathrm{H}), 4.00$ (s, 3H, $\left.\mathrm{N}-\mathrm{CH}_{3}\right)$; IR (KBr): v 3241, 1652, 1557, 1519, 1432, 1329, 1300, 1208, 1146, 1051, 827, $752 \mathrm{~cm}^{-1}$; HR-ESI-MS $m / z$ : $306.0663[\mathrm{M}+\mathrm{H}]^{+}$(calcd. for $\mathrm{C}_{12} \mathrm{H}_{9} \mathrm{~F}_{5} \mathrm{~N}_{3} \mathrm{O}, 306.0660$ ).

5-Methylisoxazol-3-yl 1-methyl-3-(trifluoromethyl)-1H-pyrazole-4-carboxylate (7bk): White powder, yield of 58.9\%, m.p. $130.7-131.1{ }^{\circ} \mathrm{C} .{ }^{1} \mathrm{H}-\mathrm{NMR}\left(\mathrm{CDCl}_{3}, 400 \mathrm{MHz}\right) \delta: 8.19$ (s, 1H, pyrazole H), 6.28 (s, $1 \mathrm{H}$, isoxazole $\mathrm{H}), 4.03\left(\mathrm{~s}, 3 \mathrm{H}, \mathrm{N}-\mathrm{CH}_{3}\right), 2.45\left(\mathrm{~s}, 3 \mathrm{H}\right.$, isoxazole $\left.\mathrm{CH}_{3}\right)$; IR $(\mathrm{KBr}): v 3290,1660,1561$, 1523, 1436, 1300, 1134, 1059, 856, 823, 711, $648 \mathrm{~cm}^{-1}$; HR-ESI-MS m/z: $276.0595[\mathrm{M}+\mathrm{H}]^{+}$(calcd. for $\left.\mathrm{C}_{10} \mathrm{H}_{9} \mathrm{~F}_{3} \mathrm{~N}_{3} \mathrm{O}_{3}, 276.0591\right)$.

\subsection{Antifungal Bioassays}

The fungicidal activity of the target Compounds $7 \mathbf{a a}-\mathbf{b k}$ were tested in vitro against the phytopathogenic fungi A. porri, $M$. coronaria, $C$. petroselini and $R$. solani using the mycelium growth rate method [26,27]. The commercially available agricultural fungicide, carbendazol, was used as the 
positive control, whereas acetone served as the negative control. The compounds were dissolved in acetone to prepare a $100-\mu \mathrm{g} / \mathrm{mL}$ stock solution for the following antifungal test.

After the mycelia were incubated at $25{ }^{\circ} \mathrm{C}$ over a certain period, the diameter of each strain was measured. The percentage inhibition was calculated as follows:

$$
I=(B-A) / B \times 100 \%
$$

where $I$ is the percentage of inhibition, $A$ is the average mycelia diameter ( $\mathrm{mm}$ ) with the compounds in Petri dishes and $B$ is the average mycelia diameter with the compounds in the blank Petri dishes.

The percentage inhibition of the compounds was determined at a dosage of $100 \mu \mathrm{g} / \mathrm{mL}$. The compounds that displayed high activity $(I>50 \%$ at $100 \mu \mathrm{g} / \mathrm{mL})$ were evaluated further at concentrations of $100,50,25,12.5,6.25$ and $0 \mu \mathrm{g} / \mathrm{mL}$. Three replicates were applied in each treatment. The $\mathrm{EC}_{50}(\mu \mathrm{g} / \mathrm{mL})$ values were estimated statistically by Probit analysis using SPSS (version 11.5) on a personal computer.

\section{Conclusions}

In conclusion, a series of novel pyrazole carboxamides and isoxazolol pyrazole carboxylates was synthesized and characterized based on the spectral data of ${ }^{1} \mathrm{H}-\mathrm{NMR}$, IR and MS in this study. The antifungal activity of the compounds was evaluated in vitro against the phytopathogenic fungi $A$. porri, M. coronaria, $C$. petroselini and $R$. solani. Among these compounds, the pyrazole carboxamides $\mathbf{7 a f}, \mathbf{7 b c}, \mathbf{7 b g}, \mathbf{7 b h}$ and $\mathbf{7 b i}$ exhibited moderate antifungal activity. The isoxazolol pyrazole carboxylate 7ai displayed strong antifungal activity against $R$. solani, with an $\mathrm{EC}_{50}$ value of $0.37 \mu \mathrm{g} / \mathrm{mL}$. This value was better than that of the commercial fungicide, carbendazol. SAR analysis results suggested that the antifungal activity of the synthesized isoxazolol pyrazole carboxylate was significantly weakened when the methyl at the C-3 of the pyrazole ring was substituted with trifluoromethyl. Thus, these novel antifungal molecules can be considered promising lead compounds with which to explore biological activity in future research.

\section{Supplementary Materials}

Supplementary materials can be accessed at: http://www.mdpi.com/1420-3049/20/03/4383/s1.

\section{Acknowledgments}

This work was supported by High Level Talents Fund of Qingdao Agricultural University (No. 6631110) and Wheat Innovation Team of Modern Agricultural Industry Technology System of Shandong Province.

\section{Author Contributions}

J.S. designed the research. J.S. and Y.Z. performed the research and analyzed the data. Y.Z. wrote the paper. All authors read and approved the final manuscript. 


\section{Conflicts of Interest}

The authors declare no conflict of interest.

\section{References}

1. Sumangala, V.; Poojary, B.; Chidananda, N.; Fernandes, J.; Kumari, N.S. Synthesis and antimicrobial activity of 1,2,3-triazoles containing Quinoline moiety. Arch. Pharm. Res. 2010, 33, 1911-1918.

2. Mert, S.; Kasımoğulları, R.; İça T.; Çolak, F.; Altun, A.; Ok, S. Synthesis, structure activity relationships, and in vitro antibacterial and antifungal activity evaluations of novel pyrazole arboxylic and dicarboxylic acid derivatives. Eur. J. Med. Chem. 2014, 78, 86-96.

3. Gawad, N.M.A.; Georgey, H.H.; Ibrahim, N.A.; Amin, N.H.; Abdelsalam, R.M. Synthesis of novel pyrazole and dihydropyrazoles derivatives as potential anti-inflammatory and analgesic agents. Arch. Pharm. Res. 2012, 35, 807-821.

4. Ouyang, G.P.; Cai, X.J.; Chen, Z.; Song, B.A.; Bhadury, P.S.; Yang, S.; Jin, L.H.; Xue, W.; Hu, D.Y.; Zeng, S. Synthesis and antiviral activities of pyrazole derivatives containing an oxime moiety. J. Agric. Food Chem. 2008, 56, 10160-10167.

5. Rangaswamy, J.; Kumar, H.V.; Harini, S.T.; Naik, N. Synthesis of benzofuran based 1,3,5-substituted pyrazole derivatives: As a new class of potent antioxidants and antimicrobials-A novel accost to amend biocompatibility. Bioorg. Med. Chem. Lett. 2012, 22, 4773-4777.

6. Hassan, G.S.; Kadry, H.H.; Abou-Seri, S.M.; Ali, M.M.; Mahmoud, A.E.E. Synthesis and in vitro cytotoxic activity of novel pyrazolo[3,4- $d$ ]pyrimidines and related pyrazole hydrazones toward breast adenocarcinoma MCF-7 cell line. Bioorg. Med. Chem. 2011, 19, 6808-6817.

7. Turan-Zitouni, G.T.; Chevallet, P.; Kiliç, F.S.; Erol, K. Synthesis of some thiazolyl-pyrazoline derivatives and preliminary investigation of their hypotensive activity. Eur. J. Med. Chem. 2000, 35, 635-641.

8. Baraldi, P.G.; Cacciari, B.; Romagnoli, R.; Spalluto, G.; Moro, S.; Klotz, K.; Leung, E.; Varani, K.; Gessi, S.; Merighi, S.; et al. Pyrazolo[4,3-e]1,2,4-triazolo[1,5-c]pyrimidine derivatives as highly potent and selective human $\mathrm{A}_{3}$ adenosine receptor antagonists: Influence of the Chain at the $\mathrm{N}^{8}$ pyrazole nitrogen. J. Med. Chem. 2000, 43, 4768-4780.

9. Lupsor, S.; Aonofriesei, F.; Iovu, M. Antibacterial activity of aminals and hemiaminals of pyrazole and imidazole. Med. Chem. Res. 2012, 21, 3035-3042.

10. Palaska, E.; Aytemir, M.; Uzbay, I.T.; Erol, D. Synthesis and antidepressant activities of some 3,5-diphenyl-2-pyrazolines. Eur. J. Med. Chem. 2001, 36, 539-543.

11. Sauter, H.; Steglich, W.; Anke, T. Strobilurins: Evolution of a new class of active substances. Angew. Chem. Int. Ed. 1999, 38, 1328-1349.

12. Shiga, Y.; Okada, I.; Ikeda, Y.; Takizawa, E.; Fukuchi, T. Insecticidal activity of $\mathrm{N}$-(4-aryloxybenzyl)pyrazole-5-carboxamides. J. Pestic. Sci. 2003, 28, 313-314.

13. Ohno, R.; Watanabe, A.; Matsukawa, T.; Ueda, T.; Sakurai, H.; Hori, M.; Hirai, K. Synthesis and herbicidal activity of new pyrazole-4-carboxamide derivatives. J. Pestic. Sci. 2004, 29, 15-26. 
14. Zhou, Y.Y.; Li, Y.X.; Li, Y.M.; Yang, X.P.; Mao, M.Z.; Li, Z.M. Design, synthesis and structure-activity of $\mathrm{N}$-glycosyl-1-pyridyl- $1 \mathrm{H}$-pyrazole-5-carboxamide as Inhibitors of Calcium channels. Chem. Res. Chin. Univ. 2013, 29, 249-255.

15. Lv, H.S.; Wang, L.Y.; Ding, X.L.; Wang, X.H.; Zhao, B.X.; Zuo, H. Synthesis and antifungal activity of novel (1-arylmethyl-3-aryl-1Hpyrazol-5-yl)(4-arylpiperazin-1-yl)methanone derivatives. J. Chem. Res. 2013, 37, 473-475.

16. Okada, I.; Okui, S.; Sekine, M.; Takahashi, Y.; Fukuchi, T. Synthesis and acaricidal activity of bicyclic pyrazole-3-carboxamide derivatives. J. Pestic. Sci. 1992, 17, 69-73.

17. Lamberth, C.; Dinges, J. Pyrazol carboxamide fungicides inhibiting succinate dehydrogenase. Bioactivity Heterocylic Compound Classes: Agrochemicals, 1st ed.; Wiley-VCH Verlag GmBH \& Co. KGaA: Weinheim, Germany, 2012; pp. 175-194.

18. Liu, F.; Wang, M.Y.; Teng, X.H.; Zhang, P.Z.; Jiang, L. Synthesis and biological evaluation of novel 2-(substituted isoxazol-4-yl)-5-arylamino-1,3,4-oxadiazoles. Res. Chem. Intermed. 2014, 40, $1575-1581$.

19. Cernuchova, P.; Vo-Thanh, G.; Milata, V.; Loupy, A. Solvent-free synthesis of quinolone derivatives. Heterocycles 2004, 64, 177-191.

20. Nishiwaki, N.; Matsushima, K.; Chatani, M.; Tamura, M.; Ariga, M. New reactivity of nitropyrimidinone: Ring transformation and N-C transfer reactions. Synlett 2004, 15, 703-707.

21. Toshio, K.; Kenji, O.; Takao, O. Preparation of 1,3-Dialkylpyrazole-4-Carboxylic Acid Esters. Jpn. Kokai Tokkyo Koho JP 2,000,044,541, 15 February 2000.

22. Kenji, H.; Bunta, W.; Osamu, K.; Seiichi, K.; Takashi, K.; Junko, S. Preparation of N-(1-Phenyl$1 H$-pyrazol-4-yl)-1H-pyrazole-4-carboxamide Derivatives as Agrochemical Fungicides. Jpn. Kokai Tokkyo Koho JP 2,010,202,649, 16 September 2010.

23. Palanki, M.S.S.; Erdman, P.E.; Gayo, F.; Leah, M.; Shevlin, G.I.; Sullivan, R.W.; Suto, M.J.; Goldman, M.E.; Ransone, L.J.; Bennett, B.L.; et al. Inhibitors of NF-אB and AP-1 gene expression: SAR studies on the pyrimidine portion of 2-chloro-4-trifluoromethyl pyrimidine-5-[N-(3',5'bis(trifluoromethyl)-phenyl) carboxamide]. J. Med. Chem. 2000, 43, 3995-4004.

24. Sun, J.L. 3-Trifluoromethyl-4-formyl Pyrazol Compounds. CN Patent 103,554,026A, 5 February 2014.

25. Sun, J.L. 3-Methyl-4-formyl Pyrazol Compounds. CN Patent 103,524,417A, 22 January 2014.

26. Xu, G.F.; Song, B.A.; Pinaki, S.B.; Yang, S.; Zhang, P.W.; Jin, L.H.; Xue, W.; Hu, D.Y.; Lu, P. Synthesis and antifungal activity of novel s-substituted-6-fluoro-4-alkyl(aryl) thioquinazoline derivatives. Bioorg. Med. Chem. 2007, 15, 3768-3774.

27. Tarun, K.C.; Prem, D.J. Antifungal activity of 4-methyl-6-alkyl-2H-pyran-2-ones. J. Agric. Food Chem. 2006, 54, 2129-2133.

Sample Availability: Samples of the compounds $\mathbf{7 a a}-\mathbf{b k}$ are available from the authors.

(C) 2015 by the authors; licensee MDPI, Basel, Switzerland. This article is an open access article distributed under the terms and conditions of the Creative Commons Attribution license (http://creativecommons.org/licenses/by/4.0/). 